(C) 2016 IEEE. Personal use of this material is permitted. Permission from IEEE must be obtained for all other uses, in any current or future media, including reprinting/republishing this material for advertising or promotional purposes, creating new collective works, for resale or redistribution to servers or lists, or reuse of any copyrighted component of this work in other works. 


\title{
Myoelectric Feature Extraction Using Temporal-Spatial Descriptors for Multifunction Prosthetic Hand Control
}

\author{
Rami N. Khushaba, Senior Member, IEEE, Ali Al-Timemy, Ahmed Al-Ani, and Adel Al-Jumaily, \\ Senior Member, IEEE
}

\begin{abstract}
We tackle the challenging problem of myoelectric prosthesis control with an improved feature extraction algorithm. The proposed algorithm correlates a set of spectral moments and their nonlinearly mapped version across the temporal and spatial domains to form accurate descriptors of muscular activity. The main processing step involves the extraction of the Electromyogram (EMG) signal power spectrum characteristics directly from the time-domain for each analysis window, a step to preserves the computational power required for the construction of spectral features. The subsequent analyses involve computing 1) the correlation between the time-domain descriptors extracted from each analysis window and a nonlinearly mapped version of it across the same EMG channel; representing the temporal evolution of the EMG signals, and 2) the correlation between the descriptors extracted from differences of all possible combinations of channels' and a nonlinearly mapped version of them, focusing on how the EMG signals from different channels correlates with each other. The proposed TemporalSpatial Descriptors (TSDs) are validated on EMG data collected from six transradial amputees performing 11 classes of finger movements. Classification results showed significant reductions (at least $8 \%$ ) in classification error rates compared to other methods.
\end{abstract}

\section{INTRODUCTION}

Myoelectric control employs Electromyogram (EMG) signals that are collected from the muscles in the residual limb to control the degrees of freedom of powered prosthetic devices worn by amputees [1]. In such a control scheme, pattern classification algorithms are usually employed to extract a representative and repeatable set of features of muscular activation, from each analysis window of the EMG data, upon which the various tasks are discriminated $[1,2]$. Previous research has shown that the success of the EMG pattern recognition system mainly depends on the quality of the extracted features, as they are of direct impact on clinical acceptance [3].

A number of feature extraction methods were utilized in the literature, these include the mean absolute value (MAV),

Rami Khushaba, Ahmed Al-Ani, and Adel Al-Jumaily are within the Faculty of Engineering and Information Technology (FEIT), University of Technology, Sydney (UTS), 15 Broadway, Ultimo 2007, NSW, Australia. (e-mail: Rami.Khushaba@uts.edu.au, Ahmed.al-ani@uts.edu.au, Adel.aljumaily@uts.edu.au).

Ali H. Al-Timemy is with the Biomedical Engineering Department, AlKhwarzmi College of Engineering, University of Baghdad, Iraq. He is also a visiting research fellow at the Centre for Robotics and Neural Systems (CRNS), Cognitive Institute, Plymouth University, UK. (e-mail: Ali.Ali@plymouth.ac.uk). The second author work is funded by the Ministry of Higher Education and Scientific Research, Iraq waveform length (WL), sloop sign changes (SSC), and number of zero crossings (ZC) [4]; fast Fourier transform (FFT) [5], wavelets and wavelet packet transform (WPT) [6, 7]; cepstral coefficients (CC), Willison amplitude (WAMP) [3]; sample entropy (ENT) [8]; and the autoregressive (AR) model parameters [9]. Phinyomark et al. compared 50 feature extraction methods for EMG pattern recognition [10]. It was found that a combination of AR coefficients and time domain features with sample entropy presented the most stable feature set. Nonetheless, despite these advancements, there are still considerable challenges in applying research outcomes to a clinically viable implementation [11]. This is partially due to a big gap between academia and industry, which limits the clinical implementation for amputees' use. Such a gap is attributed to several factors including: lack of intuitive control, poor system reliability and the lack of robustness against practical problems like limb position change, electrodes shift, varying force levels, and signal nonstationarity. Recently, the extraction of features from the first and second derivative of EMG signal was shown to produce powerful features in EMG classification [12], with results suggesting that these feature can significantly outperform several well-known features in problems with changing limb position [13], varying contraction force levels and varying forearm orientations [14].

In this paper, we extend upon our recent work in this direction [12] by adopting an approach in which we, not only extract the features from the current analysis windows of each of the individual channels, but also utilize the differences between all channel pair combinations and their nonlinear mappings. In such a case, the extracted features not only look at the temporal evolution of the EMG patterns from each EMG channel but also at the spatial relation between the power spectrum activity of the different EMG channels.

\section{Methodology}

\section{A. Signal Time-Domain Descriptors (TDD)}

The block diagram of the TDD features is shown in Fig.1, with a full description provided in [12]. Assume a sampled version of the EMG signal denoted as $x[j]$, with $j=1,2, \ldots N$, of length $N$ and a sampling frequency $f_{s}$. The EMG trace within a certain epoch can be expressed as a function of frequency $X[k]$ by means of Discrete Fourier transform (DFT). The derivations of the proposed method are based on Parseval's theorem, which states that the sum of the square of the function is equal to the sum of the square of its transform. 


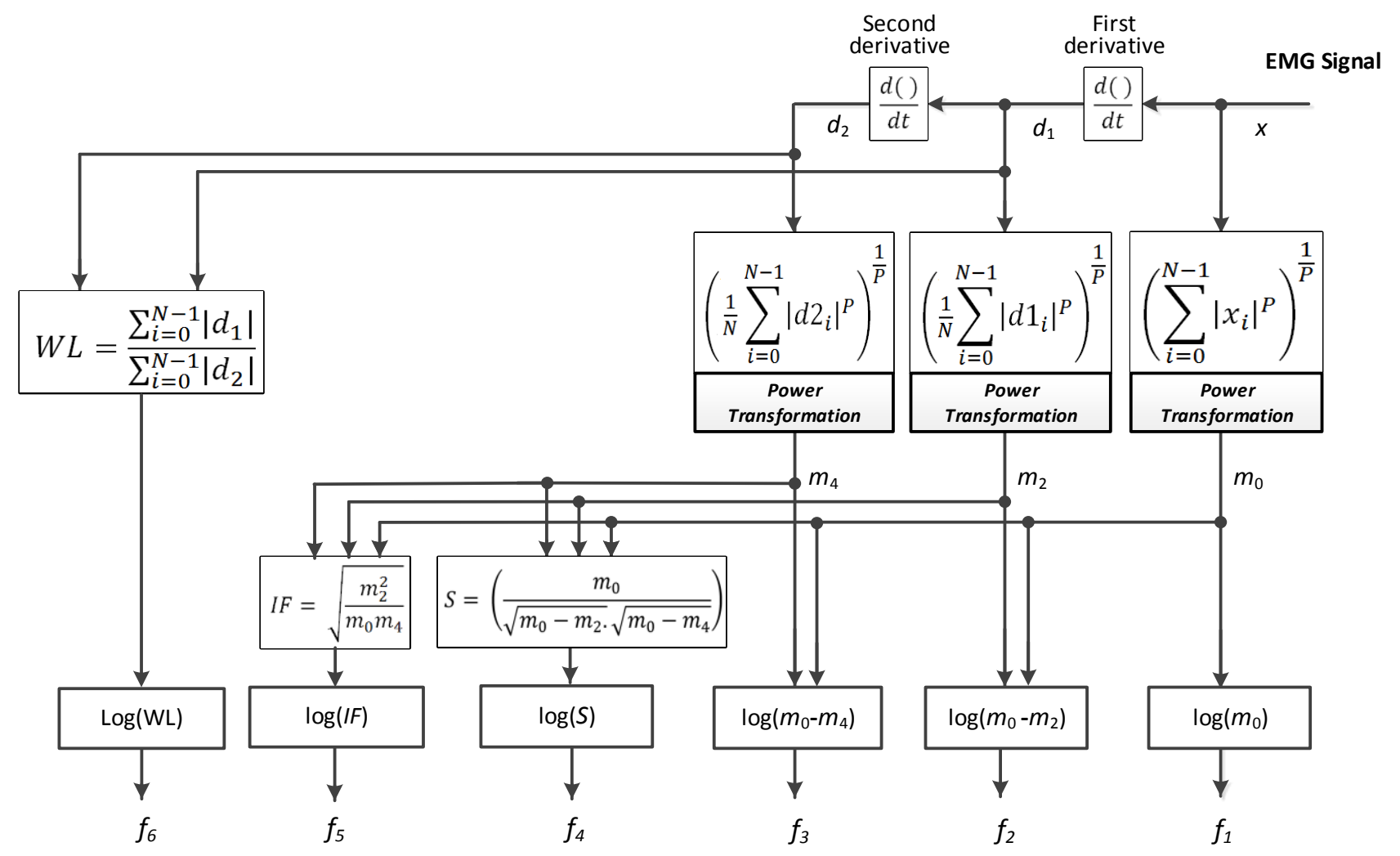

Figure 1. Block diagram for the derivation of the time-domain descriptors (TDD) feature extraction process.

$$
\sum_{\mathrm{j}=0}^{\mathrm{N}-1}|\mathrm{x}[\mathrm{j}]|^{2}=\frac{1}{\mathrm{~N}} \sum_{\mathrm{k}=0}^{\mathrm{N}-1}\left|\mathrm{X}[\mathrm{k}] \mathrm{X}^{*}[\mathrm{k}]\right|=\sum_{\mathrm{k}=0}^{\mathrm{N}-1} \mathrm{P}[\mathrm{k}]
$$

where $P[k]$ is the phase-excluded power spectrum, i.e., the result of a multiplication of $X[k]$ by its conjugate $X^{*}[k]$ divided by $N$, while $k$ is the frequency index. Thus, the first feature is simply a representative of the signal energy.

$$
\overline{\boldsymbol{m}}_{\mathbf{0}}=\sqrt{\sum_{\mathrm{j}=0}^{N-1} x[j]^{2}}
$$

The second and third features are based on the calculation of spectral moments. These were derived from the time-domain signal based on the time-differentiation property of the Fourier transform. This property simply states that the $n^{\text {th }}$ derivative of a function in the time-domain, denoted as $\Delta^{\mathrm{n}}$ for discrete time signals, is equivalent to multiplying the spectrum by $k$ raised to the $n^{\text {th }}$ power

$$
F\left[\Delta^{n} x[j]\right]=k^{n} X[k]
$$

Therefore, the second and fourth moments are defined as

$$
\begin{gathered}
\overline{\boldsymbol{m}}_{\mathbf{2}}=\sqrt{\sum_{\mathrm{k}=0}^{N-1} k^{2} P[k]}=\sqrt{\frac{1}{N} \sum_{\mathrm{k}=0}^{N-1}(k X[k])^{2}}=\sqrt{\frac{1}{N} \sum_{\mathrm{j}=0}^{N-1}(\Delta x[j])^{2}} \\
\overline{\boldsymbol{m}}_{\mathbf{4}}=\sqrt{\sum_{\mathrm{k}=0}^{N-1} k^{4} P[k]}=\sqrt{\frac{1}{N} \sum_{\mathrm{j}=0}^{N-1}\left(\Delta^{2} x[j]\right)^{2}}
\end{gathered}
$$

In this case, taking the $1^{\text {st }}$ and $2^{\text {nd }}$ derivatives of the signal reduce the total energy of the signal; hence, we square the signals before applying the derivatives and apply a power transformation to normalize the range of $m_{0}, m_{2}$, and $m_{4}$ and to reduce the effect of noise on all moment based features as follows

$$
m_{0}=\frac{\overline{\boldsymbol{m}}_{0}{ }^{\lambda}}{\lambda}, \quad m_{2}=\frac{\overline{\boldsymbol{m}}_{2}{ }^{\lambda}}{\lambda}, \quad m_{4}=\frac{\overline{\boldsymbol{m}}_{4}{ }^{\lambda}}{\lambda}
$$

With $\lambda$ empirically set to 0.1 . Once $m_{0}, m_{2}$, and $m_{4}$ are calculated, the remaining features $f_{2}$ to $f_{6}$ can be easily calculated as shown in Fig. 1 . Simply speaking, $f_{1}, f_{2}$, and $f_{3}$ are normalized versions of the power spectrum moments, $f_{4}$ is a measure of sparsity, $f_{5}$ is a measure of irregularity of the signal and $f_{6}$ is a measure of the waveform length ratios. Further details about these features are in our original article [12]. In the remaining analyses, we denote the process of extracting the six features in Fig. 1 as TDD, as we use this as a basic building block upon which the rest of the analyses depend on.

\section{B. Temporal-Spatial Correlation Features}

According to the schematic of the proposed temporalspatial descriptors (TSD) in Fig. 2, we first extract the TDD features to represent window $i$ of channel $C_{x}$ and form a vector denoted as $\mathbf{a}_{x i}=[\mathrm{a} 1, \mathrm{a} 2, \mathrm{a} 3, \mathrm{a} 4, \mathrm{a} 5, \mathrm{a} 6]$. An additional feature vector, denoted as $\mathbf{b}_{x i}=[\mathrm{b} 1, \mathrm{~b} 2, \mathrm{~b} 3, \mathrm{~b} 4, \mathrm{~b} 5, \mathrm{~b} 6]$ is then extracted from a logarithmically scaled version of the same window $\log \left(C_{x i}{ }^{2}\right)$ to end up with two feature vectors: $\mathbf{a}_{x i}$ (from the EMG record) and $\mathbf{b}_{x i}$ (from a nonlinearly scaled version of the EMG record), each made up of 6 elements. 


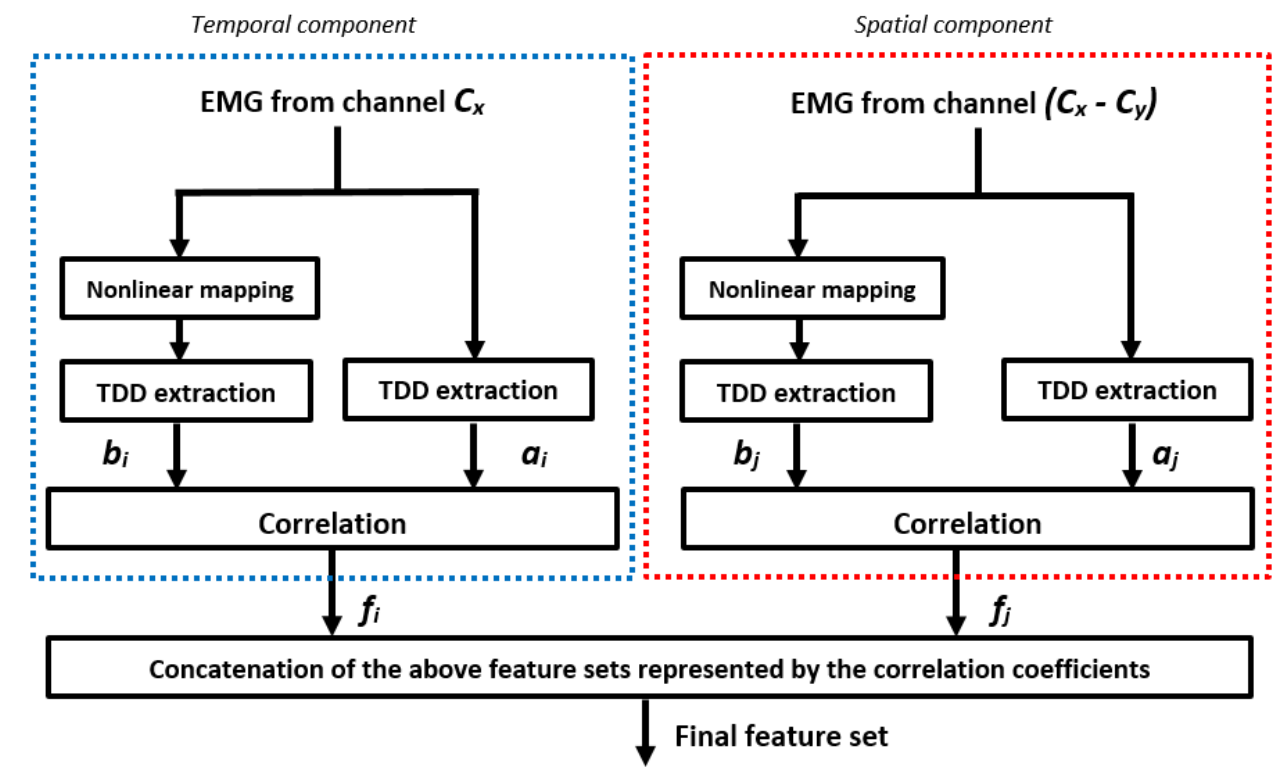

Figure 2. Block diagram of the proposed TSD feature set, which is made of a temporal component correlating TDD features extracted from each channel with those extracted from a nonlinear/smoothed version of it, and a spatial component correlating the TDD parameters from each possible channels' difference with those extracted from a nonlinear version of it.

The first part of the final feature set is obtained by calculating the element wise similarity between $\boldsymbol{a}_{x i}$ and $\boldsymbol{b}_{x i}$, i.e., made of 6 features per each EMG channel. This is achieved by calculating the cosine similarity of the two vectors given by

$$
f_{i}=\frac{-2 a_{i} b_{i}}{a_{i}{ }^{2}+b_{i}{ }^{2}}, \quad i=1,2,3, \ldots 6
$$

In a similar way, we also extracted the similarity features between the 6 TDD features from each possible difference of channels $C_{x i}-C_{y i}$ and those extracted from a nonlinear version of them, forming the second part of the final feature set. Therefore, we focus on the temporal evolution of the EMG characteristics from each channel by using a sliding window approach as well as on how the TDD from different channels relate to each other.

\section{Data Collection}

The EMG signals were originally used in [15], with 11 surface EMG sensors sampled at $2000 \mathrm{~Hz}$ with a custombuilt data acquisition system (NI USB 6210, National Instruments, USA) with 16-bit resolution. A Virtual Instrument (VI) was developed in LABVIEW (National Instruments, USA) to display and store the EMG signals. The data from six transradial amputees (TR1 to TR6) persons were collected at the artificial limbs and rehabilitation center in Baghdad and Babylon. Eleven classes of finger movements were carried out by each amputee, including: little flexion (f1), ring flexion (f2), middle flexion (f3), index flexion (f4), rest position, little extension (e1), ring extension (e2), middle extension (e3), index extension (e4), thumb flexion (f5), and thumb extension (e5). During the recording of the EMG signals, each participant sat on a chair in front of a computer with the Labview interface screen to view all the EMG sensors in real-time, while imagining the movements. Six trails of each movement were conducted. Ethical approval was obtained from the Human Ethics Committee of the Faculty of Science and Technology at Plymouth
University. All Subjects gave their written informed consent to participate in the study.

\section{EMG Pattern Recognition Analysis}

In the experiments, an overlapping windowing scheme was utilized to extract the proposed features, with an analysis window size of $150 \mathrm{~ms}$ and an increment of $75 \mathrm{~ms}$. MATLAB® 2015b software (Mathworks, USA) was used to perform the analyses. To evaluate the performance of the proposed feature set, denoted as Temporal-Spatial Descriptors (TSD), we compare the achieved classification performance against the classification performance of some well-known EMG feature extraction methods from the literature. These include: a combination of Autoregressive model parameters (5th order) with signal root mean square denoted as AR-RMS [16]; the combination of MAV, ZC, SSC, WL denoted as TD feature set [4]; the same combination with the WAMP features denoted as TD1 feature set; and the wavelet features extraction method, utilizing the mean of the squared wavelet coefficients at each level as features, with Symmlet family of wavelets and 5 decomposition levels, denoted as Wavelet; and our original TDPSD feature extraction method [12]. The dimensionality of all of the extracted feature sets was reduced using the Spectral Regression (SR) dimensionality reduction method proposed by Cai et al. [17]. SR maps the original feature set into a new domain with $\mathrm{c}-1$ features only, with $\mathrm{c}$ being the number of classes, i.e., 10 features in this problem. Finally, in terms of classification accuracy, we have utilized a Linear Discriminant Analysis (LDA) classifier with quadratic function as well as the $k$-Nearest Neighbour $(k N N)$ classifier, with $\mathrm{k}=1$, which was chosen empirically. In all analyses, features extracted from the first trial of each hand movement were assigned to the training set, while features extracted from the remaining trials were assigned to the testing set. This would in turn make the classification task more challenging for any feature extraction method due to the small size of the training feature set. 


\section{RESULTS AND DISCUSSION}

The average classification error rates across all of the six amputees are shown in Fig. 3 for all of the utilized feature extraction methods across the two classifiers of LDA and $k N N$. These results clearly depict few important points, the first is that the error rates achieved by the proposed TSD feature extraction method were much lower than that achieved by all other methods across both classifiers. An analysis of variance tests with a significance level of 0.05 revealed significant differences between TSD and the remaining methods ( $p$-value $<0.01$ for all tests). Secondly, there were no significant differences between the performances of the two different classifiers for the different feature extraction methods. This is an important property, which reflects the fact that the extracted features were robust against the change of the classifier.

Finally, it is important to mention here that TDPSD which we presented earlier in [12] is only focusing on the temporal part of the feature extraction and that it does not look at the relation between the EMG signals from the different channels. In comparison to TDPSD, and all other feature extraction methods, our new TSD method also looks at how the EMG power spectrum characteristics of the different channels change across the different movement and was therefore more capable of further reducing the classification error rates. This in turn proves our main point of extending the feature extraction process to look at the temporal and spatial evolution of the EMG signals from different channels when performing different hand movements.

\section{CONCLUSION}

A new feature extraction method for EMG pattern recognition was presented based on time domain descriptors that utilized spatial and temporal correlations. The method proposed to extract a set of features that are equivalent to the power spectrum moments directly from the time-domain by utilizing Fourier relations and Parseval's theorem. In the proposed method, the TSD descriptors were extracted from each analysis window and a nonlinearly mapped version of it along each channel to reflect the temporal component and then along differences of the various channel combinations to reflect the spatial component. The resulting algorithm gave

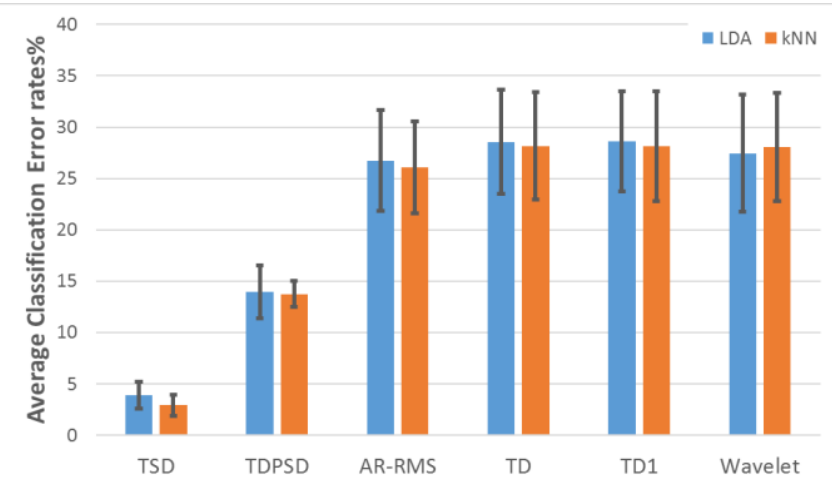

Figure 3. Average classification error rates across all amputees, with bars representing standard deviation. significant reductions in classification error rates on various EMG datasets collected from six transradial amputee subjects. Our results revealed that there were no significant differences between the error rates achieved by using the classifiers of LDA and $k \mathrm{NN}$.

\section{REFERENCES}

[1] D. Farina, N. Jiang, H. Rehbaum, A. Holobar, B. Graimann, H. Dietl, et al., "The extraction of neural information from the surface EMG for the control of upper-limb prostheses: emerging avenues and challenges," IEEE Trans Neural Syst Rehabil Eng, vol. 22, pp. 797809, Jul 2014.

[2] E. Scheme and K. Englehart, "Electromyogram pattern recognition for control of powered upper-limb prostheses: state of the art and challenges for clinical use," J Rehabil Res Dev, vol. 48, pp. 643-59, 2011 .

[3] M. Zardoshti-Kermani, B. C. Wheeler, K. Badie, and R. M. Hashemi, "EMG feature evaluation for movement control of upper extremity prostheses," IEEE Trans on Rehab Eng, vol. 3, pp. 324-333, 1995.

[4] B. Hudgins, P. Parker, and R. Scott, "A new strategy for multifunction myoelectric control," IEEE Trans Biomed Eng, vol. 40, 1993.

[5] J. He, D. Zhang, X. Sheng, S. Li, and X. Zhu, "Invariant Surface EMG Feature Against Varying Contraction Level for Myoelectric Control Based on Muscle Coordination," IEEE J Biomed Health Inform, vol. 19, pp. 874-82, May 2015.

[6] K. Englehart, B. Hudgins, and P. A. Parker, "A wavelet-based continuous classification scheme for multifunction myoelectric control," IEEE Trans Biomed Eng, vol. 48, pp. 302-11, Mar 2001.

[7] R. N. Khushaba, A. Al-Jumaily, and A. Al-Ani, "Novel feature extraction method based on fuzzy entropy and wavelet packet transform for myoelectric Control," in Communications and Information Technologies, 2007. ISCIT '07. International Symposium on, 2007, pp. 352-357.

[8] X. Zhang and P. Zhou, "Sample entropy analysis of surface EMG for improved muscle activity onset detection against spurious background spikes," Journal of Electromyography and Kinesiology, vol. 22, pp. 901-907, 2012.

[9] Y. Huang, K. B. Englehart, B. Hudgins, and A. D. Chan, "A Gaussian mixture model based classification scheme for myoelectric control of powered upper limb prostheses," IEEE Trans Biomed Eng, vol. 52, pp. 1801-11, Nov 2005.

[10] A. Phinyomark, F. Quaine, S. Charbonnier, C. Serviere, F. TarpinBernard, and Y. Laurillau, "EMG feature evaluation for improving myoelectric pattern recognition robustness," Expert Systems with Applications, vol. 40, pp. 4832-4840, 2013.

[11] N. Jiang, S. Dosen, K. R. Muller, and D. Farina, "Myoelectric control of artificial limbs- is there a need to change focus?," IEEE Signal Processing Magazine, vol. 29, pp. 148-152, 2012.

[12] A. Al-Timemy, R. Khushaba, G. Bugmann, and J. Escudero, "Improving the Performance Against Force Variation of EMG Controlled Multifunctional Upper-Limb Prostheses for Transradial Amputees," IEEE Trans Neural Syst Rehabil Eng, Jun 232015.

[13] R. N. Khushaba, M. Takruri, J. V. Miro, and S. Kodagoda, "Towards limb position invariant myoelectric pattern recognition using timedependent spectral features," Neural Networks, vol. 55, pp. 42-58, 2014.

[14] R. N. Khushaba, A. Al-Timemy, and S. Kodagoda, "Influence of multiple dynamic factors on the performance of myoelectric pattern recognition," in Engineering in Medicine and Biology Society (EMBC), 2015 37th Annual International Conference of the IEEE, 2015, pp. 1679-1682.

[15] A. H. Al-Timemy, G. Bugmann, J. Escudero, and N. Outram, "Classification of Finger Movements for the Dexterous Hand Prosthesis Control With Surface Electromyography," IEEE Journal of Biomedical and Health Informatics, vol. 17, pp. 608-618, 2013.

[16] A. Chan and G. Green, "Myoelectric control development toolbox," in Conference of the Canadian Medical \& Biological Engineering Society, ed, 2007.

[17] D. Cai, X. He, and J. Han, "SRDA: An Efficient Algorithm for Large-Scale Discriminant Analysis," IEEE Trans. Knowl. Data Eng., vol. 20, pp. 1-12, 2008 . 